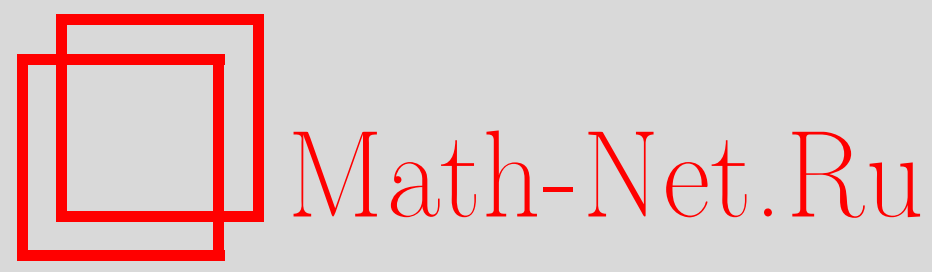

А. А. Туганбаев, Модули, у которых все подмодули являются аннуляторами, Дискрет. матем., 2012, том 24, выпуск $3,39-50$

DOI: https://doi.org/10.4213/dm1195

Использование Общероссийского математического портала Math-Net.Ru подразумевает, что вы прочитали и согласны с пользовательским соглашением http://www . mathnet.ru/rus/agreement

Параметры загрузки:

IP : 54.198 .67 .100

26 апреля 2023 г., 02:56:05 


\title{
Модули, у которых все подмодули являются аннуляторами
}

\author{
(c) 2012 г. А. А. Туганбаев
}

\begin{abstract}
Исследуются комультипликационные модули над не обязательно коммутативными кольцами.

Данная работа поддержана Российским фондом фундаментальных исследований, проект 08-01-00693-а: «Структурная теория колец».
\end{abstract}

Все кольца предполагаются ассоциативными и с ненулевой единицей, а модули унитарными. Выражение, что $A$ есть инвариантное кольцо, означает, что $A$ - инвариантное справа и слева кольцо. Если $A-$ кольцо и $M-$ правый $A$-модуль, то для любого подмножества $B$ в $A$ через $\ell_{M}(B)$ обозначается левый аннулятор $\{m \in M \mid m B=0\}$ для $B$ в $M$. Модуль $M_{A}$ называется комультипликационным, если для любого его подмодуля $X$ существует такой идеал $B$ кольца $A$, что $X=\ell_{M}(B)$. Например, все циклические модули над кольцами вычетов или групповыми алгебрами конечных циклических групп над конечными полями являются конечными комультипликационными модулями. Комультипликационные модули изучались во многих работах (см., например, [1-12]). В этих работах в основном изучаются комультипликационные модули над коммутативными кольцами.

Если $A$ - коммутативное кольцо и $M-$ ненулевой $A$-модуль, то известны приведенные ниже результаты I-III.

(I) Если $M$ - комультипликационный модуль, то $M$ - существенное расширение прямой суммы попарно неизоморфных простых модулей, причем произвольный ненулевой конечнопорожденный подмодуль $X$ в $M$ является существенным расширением конечной прямой суммы попарно неизоморфных простых модулей и кольцо $A / r_{A}(X)$ имеет только конечное число максимальных идеалов.

(II) $M-$ комультипликационный полупримитивный модуль

$\Longleftrightarrow M-$ комультипликационный полупростой модуль

$\Longleftrightarrow$ либо $M$ прост, либо

$$
M=\bigoplus_{i \in I} M_{i}, \quad|I|>1,
$$

где все $M_{i}-$ попарно неизоморфные простые модули и

$$
\bigcap_{j \neq i} r_{A}\left(M_{j}\right) \nsubseteq r_{A}\left(M_{i}\right)
$$

для каждого $i \in I$. 
(III) Если кольцо $A$ регулярно, то $M$ является комультипликационным в точности тогда, когда либо $M$ прост, либо

$$
M=\bigoplus_{i \in I} M_{i}, \quad|I|>1,
$$

где все $M_{i}$ - попарно неизоморфные простые модули и

$$
\bigcap_{j \neq i} r_{A}\left(M_{j}\right) \not r_{A}\left(M_{i}\right)
$$

для каждого $i \in I$.

В связи с известными утверждениями I-III мы докажем теорему 1, которая является основным результатом работы.

Теорема 1. Пусть $A-$ - кольц̧о с коммутативным умножением правых идеалов и $M-$ ненулевой правый А-модуль.

(1) Если $M$ - комультипликационный модуль, то $M$ - существенное расширение прямой суммы попарно неизоморфных простых модулей, причем произвольный ненулевой конечнопорожденный подмодуль $X$ в $M$ является существенным расширением конечной прямой суммы попарно неизоморфных простых модулей $X_{1}, \ldots, X_{n}, u$ кольцо $A / r_{A}(X)$ имеет только конечное число максимальных правых идеалов.

(2) М является комультипликационным и полупримитивным модулем в точности тогда, когда либо $М$ прост, либо

$$
M=\bigoplus_{i \in I} M_{i}, \quad|I|>1,
$$

где все $M_{i}$ - попарно неизоморфные простые модули и

$$
\bigcap_{j \neq i} r_{A}\left(M_{j}\right) \nsubseteq r_{A}\left(M_{i}\right)
$$

для каждого $i \in I$.

(3) Если кольияо А регулярно, то М является комультипликационным в точности тогда, когда либо $M$ прост, либо

$$
M=\bigoplus_{i \in I} M_{i}, \quad|I|>1,
$$

где все $M_{i}$ - попарно неизоморфные простые модули и

$$
\bigcap_{j \neq i} r_{A}\left(M_{j}\right) \nsubseteq r_{A}\left(M_{i}\right)
$$

для каждого $i \in I$.

Кольцо $A$ называется кольцом с коммутативным умножением правых идеалов, если $B C=C B$ для любых правых идеалов $B$ и $C$ кольца $A$; такие кольца инвариантны справа, 
поскольку можно взять $C=A$ и получить $B=A B$. Напомним, что кольцо называется инвариантным справа, если все его правые идеалы являются идеалами. Заметим, что существуют инвариантные справа кольца с некоммутативным умножением правых идеалов (см., например, пример 9.10 в [14]). Кроме того, класс всех колец с коммутативным умножением правых идеалов включает в себя все коммутативные кольца, кольца формальных степенных рядов от одной переменной над телами, все факторкольца любых прямых произведений тел и все строго регулярные кольца. Напомним, что кольцо $A$ называется регулярным (строго регулярным), если каждый его главный правый или левый идеал порождается идемпотентом (центральным идемпотентом).

Доказательство теоремы 1 разобьем на ряд утверждений; некоторые из них представляют самостоятельный интерес. Вначале приведем необходимые определения и обозначения. Через $J(M)$ обозначается радикал Джекобсона модуля $M$. Модуль $M$ называется полупримитивным, если $J(M)=0$.

Лемма 1. Если $A-$-инариантное справа кольц̧о и $B-$ правый идеал в $A$, то

$$
B(1-b) \subseteq(1-b) B
$$

для любого элемента $b \in B$.

Доказательство. Пусть $b_{1} \in B$. Так как кольцо $A$ инвариантно справа, то $(1-b) A-$ идеал. Поэтому

$$
b_{1}(1-b)=(1-b) a
$$

для некоторого элемента $a \in A$. Тогда

$$
a=(1-b) a+b a=b_{1}(1-b)+b a \in B, \quad B(1-b) \subseteq(1-b) B .
$$

Лемма 2. Пусть $A-$-инариантное справа кольияо и $M-$ конечнопорожденный правый $A$-модуль. Если $B-$ правый идеал в $A$ и $M=M B$, то $M(1-b)=0$ для некоторого элемента $b \in B$.

Доказательство. Пусть

$$
M=\sum_{i=1}^{n} m_{i} A .
$$

Проведем индукцию по $n$.

Допустим, что $n=1$ и $M=m_{1} A$. Обозначим $m=m_{1}$. Так как кольцо $A$ инвариантно справа, то

$$
r(M)=r(m), \quad m A=m A B=m B .
$$

Поэтому $m(1-b)=0$ для некоторого элемента $b \in B$. Тогда $M(1-b)=0$.

Допустим, что $n \geqslant 2$ и утверждение верно для всех натуральных чисел, меньших $n$. Обозначим

$$
N=\sum_{i=1}^{n-1} m_{i} A .
$$


Сначала докажем, что

$$
A=B+\left(m_{n} A^{\cdot} \cdot N\right), \quad N=N B .
$$

Положим $Y=0$ для $n=2$ и

$$
Y=\sum_{i=2}^{n-1} m_{i} A
$$

для $n \geqslant 3$.

Для каждого $m_{i}$ существует такой элемент $b_{i} \in B$, что

$$
m_{i} \in M=M B=\sum_{j=1}^{n} m_{j} B, \quad m_{i}\left(1-b_{i}\right) \in \sum_{j \neq i} m_{j} B .
$$

Тогда

$$
m_{n} A\left(1-b_{n}\right) \subseteq N B=m_{1} B+Y B, \quad m_{1} A\left(1-b_{1}\right) \in Y B+m_{n} B,
$$

поскольку $A$ инвариантно справа. Кроме того, $B\left(1-b_{1}\right) \subseteq\left(1-b_{1}\right) B$ по лемме 1 . Поэтому

$$
A=B+\left(m_{n} A \cdot N\right)
$$

И

$$
\begin{aligned}
m_{1} A\left(1-b_{1}\right)\left(1-b_{n}\right) & \subseteq\left(Y B+m_{n} B\right)\left(1-b_{n}\right) \\
& \subseteq Y B+m_{n} B\left(1-b_{n}\right) \\
& \subseteq Y B+m_{n}\left(1-b_{n}\right) B \\
& \subseteq Y B+m_{1} B=N B .
\end{aligned}
$$

Тогда существует такой элемент $b^{*} \in B$, что

$$
m_{1}\left(1-b_{1}\right)\left(1-b_{n}\right)-m_{1} b^{*} \in Y B, \quad m_{1}\left[\left(1-b_{1}\right)\left(1-b_{n}\right)-b^{*}\right] \in Y B .
$$

Обозначим

$$
b=1-\left[\left(1-b_{1}\right)\left(1-b_{n}\right)-b^{*}\right] \in B .
$$

Тогда

$$
\begin{aligned}
m_{1} & =m_{1} b+m_{1}(1-b) \\
& =m_{1} b+m_{1}\left[\left(1-b_{1}\right)\left(1-b_{n}\right)-b^{*}\right] \in m_{1} B+Y B=N B .
\end{aligned}
$$

Поэтому $m_{1} A \subseteq N B$. Аналогично можно доказать, что $m_{i} A \subseteq N B$ при $i=2, \ldots, n-1$. Поэтому $N=N B$.

Таким образом, доказано, что

$$
A=B+\left(m_{n} A^{\circ} \cdot N\right), \quad N=N B .
$$

Кроме того,

$$
A=B+r(N)
$$


по предположению индукции. Тогда

$$
\begin{aligned}
M\left(m_{n} A^{\cdot} \cdot N\right) r(N) & =\left(N+m_{n} A\right)\left(m_{n} A^{\bullet} \cdot N\right) r(N) \\
& =N\left(m_{n} A^{\cdot} \cdot N\right) r(N)+m_{n} A\left(m_{n} A^{\bullet} . N\right) r(N) \subseteq N r(N)=0 .
\end{aligned}
$$

Поэтому

$$
\left(m_{n} A^{\cdot} \cdot N\right) r(N) \subseteq r(M)
$$

и

$$
\begin{aligned}
A & =A \cdot A=\left(B+\left(m_{n} A^{\cdot} \cdot N\right)\right)(B+r(N)) \\
& \subseteq B+\left(m_{n} A^{\cdot} . N\right) r(N) \subseteq B+r(M) \subseteq A .
\end{aligned}
$$

Тогда $A=B+r(M)$ и $M(1-b)=0$ для некоторого элемента $b \in B$. Лемма доказана.

Следующие два утверждения известны и могут быть легко проверены непосредственHо.

Лемма 3 ([1], лемма 1.2). Пусть $A-$ кольцуо, $B$ - идеал в $A$ и $M-$ правыцй $A$-модуль, удовлетворяюший условию $M B=0$. Тогда $M-$ комультипликационный $A$-модуль тогда и только тогда, когда $M$ - комультипликационньй $A / B$-модуль.

Лемма 4 ([2], теорема 3.17, лемма 3.7). Пусть $A$ - кольц̧о и $M$ - правый $A$-модуль. Равносильнь следующие условия:

(1) М - комультипликационный модуль;

(2) $X=\ell_{M}\left(r_{A}(X)\right)$ для каждого подмодуля $X$ модуля $M$;

(3) каждый подмодуль модуля $M$ - комультипликащионньй модуль.

Следующая лемма фактически хорошо известна $[1,2]$. Для удобства приведем это утверждение с доказательством.

Лемма 5. Пусть $A-$ кольияо и $M$ - комультипликационный правый $A$-модуль. Если $X-$ ненулевой подмодуль в $M$, то $X$ - комультипликационный $A / r_{A}(X)$-модуль $u f(X) \subseteq X$ для любого гомоморфизма $f: X \rightarrow M . B$ частности, $M$ не содержит прямую сумму двух изоморфных ненулевых модулей.

Доказательство. По лемме 3 и лемме $4, X$ - комультипликационный $A / r_{A}(X)$-модуль. Так как $M-$ комультипликационный модуль, то $X=r_{M}(B)$ для некоторого идеала $B$ в A. Тогда

$$
f(X) B \subseteq f(X B)=f(0)=0,
$$

откуда

$$
f(X) \subseteq r_{M}(B)=X
$$

Для коммутативных колец приведенная ниже лемма была доказана в [3] (предложение 3.1 (b)-(c)). 
Лемма 6. Пусть $A-$ кольц̧о, $M$ - комультипликационный правый $A$-модуль и $B-$ такой идеал кольц̧а $A$, что $\ell_{M}(B)=0 u B C=C B$ для любого идеала $C$ кольцуа $A$. Тогда справедливы следующие утверждения.

(1) Верно соотношение $M=M B$.

(2) Для любого элемента $m \in M$ существует элемент $b \in B$, удовлетворяющий условию $m(1-b)=0$.

Доказательство. Докажем первую часть. Так как $M-$ комультипликационный модуль, то $M B=\ell_{M}(C)$ для некоторого идеала $C$ кольца $A$. Тогда

$$
\begin{aligned}
M C B=M B C=\ell_{M}(C) C & =0, \\
M C & \subseteq \ell_{M}(B)=0, \\
M & =l_{M}(C)=M B .
\end{aligned}
$$

Докажем вторую часть леммы. Так как $\ell_{M}(B)=0$, то $\ell_{m A}(B)=0$. Кроме того, $m A$ - комультипликационный модуль в силу леммы 3 . Ввиду только что доказанной первой части леммы, $m A=m A B=m B$. Поэтому существует элемент $b \in B$, удовлетворяющий условию $m(1-b)=0$.

Лемма 7 ([13], теорема 9.4.3). Модуль М является существенным расширением конечной прямой суммы простых модулей тогда и только тогда, когда для любого множества $\left\{P_{i}\right\}_{i \in I}$ подмодулей $P_{i}$ в $X$, удовлетворяющих условию

$$
\bigcap_{i \in I} P_{i}=0,
$$

существует конечное подмножество $J$ в I, удовлетворяющее условию

$$
\bigcap_{j \in J} P_{j}=0 .
$$

Лемма 8. Для ненулевого модуля М равносильны следующие условия:

(1) $M$ полупримитивен и каждый ненулевой цчиклический подмодуль в $М$ является существенным расширением конечной прямой суммы простых модулей;

(2) модуль $M$ полупрост.

Доказательство. Импликация (2) $\Rightarrow(1)$ проверяется непосредственно.

Для доказательства справедливости импликации $(1) \Rightarrow(2)$ достаточно доказать, что произвольный циклический подмодуль $X$ модуля $M$ изоморфен подмодулю полупростого модуля. Так как модуль $M$ полупримитивен, то

$$
\bigcap_{i \in I} P_{i}=0,
$$

где $\left\{P_{i}\right\}_{i \in I}-$ множество всех максимальных подмодулей в $M$. Обозначим

$$
P_{i}=X \cap Q_{i}, \quad i \in I .
$$


Тогда

$$
\bigcap_{i \in I} Q_{i}=0
$$

где для любого $i \in I$ модуль $X / Q_{i}$ либо прост, либо равен нулю. По лемме 7 , существует конечное подмножество $J$ в $I$, удовлетворяющее условию

$$
\bigcap_{j \in J} Q_{j}=0
$$

Ясно, что модуль $X$ изоморфен подмодулю полупростого модуля $\bigoplus_{j \in J}\left(X / Q_{j}\right)$.

Лемма 9. Пусть $A-$ кольцฺо, $M$ - комультипликационный правый $A$-модуль и $M$ содержит прямую сумму ненулевых модулей $X_{i}$, где $i \in I \quad u|I| \geqslant 2$. Тогда

$$
\bigcap_{j \neq i} r_{A}\left(X_{j}\right) \nsubseteq r_{A}\left(X_{i}\right)
$$

для любого $i \in I$, причем $X_{i} \not X_{i}$ при $i \neq j$.

Доказательство. Обозначим

$$
X=\bigoplus_{i \in I} X_{i}, \quad Y_{i}=\bigoplus_{j \neq i} X_{j}
$$

Допустим, что

$$
\bigcap_{j \neq i} r_{A}\left(X_{j}\right) \subseteq r_{A}\left(X_{i}\right)
$$

для некоторого $i \in I$. По лемме $4, X$ - комультипликационный модуль и

$$
Y_{i}=\ell_{X}\left(r_{A}\left(Y_{i}\right)\right)=\ell_{X}\left(\bigcap_{j \neq i} r_{A}\left(X_{j}\right)\right) \subseteq \ell_{X}\left(r_{A}\left(X_{i}\right)\right)=X_{i}
$$

Поэтому

$$
0 \neq Y_{i}=Y_{i} \cap X_{i}=0
$$

и получаем противоречие.

Лемма 10. Пусть $A-$ кольйо и $P-$ такой максимальный правый идеал в $A$, что $P-$ идеал. Тогда справедливы следующие утверждения.

(1) Факторкольичо $A / P$ является телом, $A / P-$ простой правый $A$-модуль и $P=Q$ для любого такого максимального правого идеала $Q$, что $(A / P)_{A} \cong(A / Q)_{A}$.

(2) Если $M$ - комультипликационный правый $A$-модуль и $\ell_{M}(P) \neq 0$, то $\ell_{M}(P)-$ простой модуль. 
Доказательство. Докажем первое утверждение. Так как $P$ - идеал и $P-$ максимальный правый идеал, то факторкольцо $A / P$ является телом и $A / P$ - простой правый $A$-модуль.

Допустим, что $P \neq Q$. Тогда $A=P+Q$, и существует $A$-модульный изоморфизм $P /(P \cap Q) \rightarrow Q /(P \cap Q)$. Так как $A=P+Q$, то этот изоморфизм продолжается до гомоморфизма $\bar{f}: A /(P \cap Q) \rightarrow Q /(P \cap Q)$ с ядром $Q /(P \cap Q)$. Так как модуль $A_{A}$ проективен, то $\bar{f}$ поднимается до такого гомоморфизма $f: A_{A} \rightarrow A_{A}$, что $f(A) \subseteq Q$, $f(Q) \subseteq P \cap Q$, и $f(P) \nsubseteq P \cap Q$. Обозначим $a=f(1) \in A$. Тогда

$$
f(P)=a P \subseteq P \cap Q,
$$

поскольку $P$ - идеал. Получено противоречие.

Докажем второе утверждение. Так как факторкольцо $A / P$ является телом, то ненулевой правый $A / P$-модуль $\ell_{M}(P)$ есть прямая сумма изоморфных простых модулей. По лемме 5 , модуль $\ell_{M}(P)$ прост.

Лемма 11. Пусть $A-$ кольио, в котором все максимальные правые идеаль являются идеалами, и пусть $\left\{X_{i}\right\}_{i \in I}-$ некоторое множество простых правых $A$-модулей, $|I| \geqslant 2$. Равносильны следуюшие условия:

(1) $\bigoplus_{i \in I} X_{i}-$ комультипликачионный правый А-модуль;

(2) $\bigcap_{j \neq i} r_{A}\left(X_{j}\right) \nsubseteq r_{A}\left(X_{i}\right)$ для любого $i \in I$, причем $X_{i} ¥ X_{j}$ при $i \neq j$.

Доказательство. Импликация (1) $\Rightarrow$ (2) следует из леммы 9.

Докажем справедливость импликации (2) $\Rightarrow(1)$. Обозначим

$$
X=\bigoplus_{i \in I} X_{i}, \quad P_{i}=r_{A}\left(X_{i}\right) .
$$

Из условия следует, что все факторкольца $A / P_{i}$ являются телами. Пусть $N-$ ненулевой собственный подмодуль в $X$. Так как $X-$ прямая сумма попарно неизоморфных простых модулей $X_{i}$, то найдется такое собственное непустое подмножество $J$ в $I$, что

$$
N=\bigoplus_{j \in J} X_{j}
$$

Тогда

$$
r_{A}(N)=\bigcap_{j \in J} P_{j} .
$$

В силу леммы 4, достаточно доказать, что

$$
N=\ell_{X}\left(r_{A}(N)\right) .
$$

Допустим, что $N \neq \ell_{X}\left(r_{A}(N)\right)$. Тогда $\ell_{X}\left(r_{A}(N)\right)$ строго содержит $N$. Кроме того, $X$ - прямая сумма неизоморфных простых модулей. Поэтому $\ell_{X}\left(r_{A}(N)\right)$ содержит модуль $X_{i}$ для некоторого $i \in I \backslash J$. Тогда $X_{i} r_{A}(N)=0$. Поэтому $r_{A}(N) \subseteq P_{i}$. Кроме того,

$$
\bigcap_{j \neq i} P_{j} \subseteq \bigcap_{j \in J} P_{j}=r_{A}(N) \subseteq P_{i} .
$$


По условию,

$$
\bigcap_{j \neq i} P_{j} \nsubseteq P_{i}
$$

Получено противоречие.

Лемма 12. Пусть $A-$ кольияо с коммутативным умножением правых идеалов, $M$ ненулевой комультипликационный правый $A$-модуль, $X$ - ненулевой конечнопорожденный подмодуль в М. Тогда кольичо А инвариантно справа, причем верны утверждения ниже.

(1) Если $B-$ - деал кольц̧а $A$, удовлетворяющчй условию $\ell_{M}(B)=0$, то существует элемент $b \in B$, удовлетворяющий условию $X(1-b)=0$.

(2) Если $\left\{M_{i}\right\}_{i \in I}-$ некоторое множество подмодулей в $M$, удовлетворяющих условию

$$
\bigcap_{i \in I} M_{i}=0
$$

$u$

$$
B=\sum_{i \in I} r_{A}\left(M_{i}\right)
$$

то существуют конечное подмножество $J \subseteq I$ илемент $b \in \sum_{j \in J} r_{A}\left(M_{i}\right) \subseteq B$, удовлетворяющие условию $X(1-b)=0$.

(3) Если подмодуль $X$ точен, то $\ell_{X}(B) \neq 0$ для любого собственного идеала $B$ кольuза $A$.

(4) X - существенное расширение конечной прямой суммы попарно неизоморфных простых модулей $X_{1}, \ldots, X_{n}$, и любой простой подмодуль в $X$ совпадает с одним из модулей $X_{1}, \ldots, X_{n}$.

(5) М - существенное расширение прямой суммы попарно неизоморфных простых модулей.

(6) Кольцо $A / r_{A}(X)$ имеет только конечное число максимальных правых идеалов.

Доказательство. В силу леммы $3, X$ - комультипликационный модуль. Заметим также, что кольцо $A$ инвариантно справа.

Докажем первое утверждение. Так как

$$
\ell_{X}(B) \subseteq \ell_{M}(B)=0,
$$

TO

$$
X=X B
$$

в силу части 1 леммы 6. В силу леммы 2 ,

$$
X(1-b)=0
$$

для некоторого элемента $b \in B$. 
Докажем второе утверждение. В силу леммы 4,

$$
M_{i}=\ell_{M}\left(r_{A}\left(M_{i}\right)\right)
$$

для каждого $i \in I$. Тогда

$$
0=\bigcap_{i \in I} M_{i}=\bigcap_{i \in I} \ell_{M}\left(r_{A}\left(M_{i}\right)\right)=\ell_{M}\left(\sum_{i \in I} r_{A}\left(M_{i}\right)\right)=\ell_{M}(B) .
$$

В силу только что доказанного утверждения 1 , существует элемент $b \in B$, удовлетворяющий условию $X(1-b)=0$. Тогда

$$
1-b \in r_{A}(X)+\sum_{i \in I} r_{A}\left(M_{i}\right.
$$

Поэтому существует конечное подмножество $J \subseteq I$, удовлетворяющее условию

$$
b \in \sum_{j \in J} r_{A}\left(M_{i}\right) \subseteq B .
$$

Докажем третье утверждение. Допустим, что $\ell_{X}(B)=0$ для некоторого собственного идеала $B$ кольца $A$. В силу только что доказанного утверждения 1 , существует элемент $b \in B$, удовлетворяющий условию $1-b \in r_{A}(X)=0$. Тогда $1=b \in B$ и $B=A$; получено противоречие.

Докажем четвертое утверждение. Без ограничения общности можно считать, что модуль $X$ точен. По лемме $5, X$ не содержит прямую сумму двух изоморфных ненулевых модулей. Поэтому достаточно доказать, что $X$ - существенное расширение конечной прямой суммы простых модулей. По лемме 7, достаточно доказать, что для любого множества $\left\{X_{i}\right\}_{i \in I}$ подмодулей $X_{i}$ в $X$, удовлетворяющих условию

$$
\bigcap_{i \in I} X_{i}=0
$$

существует конечное подмножество $J$ в $I$, удовлетворяющее условию

$$
\bigcap_{j \in J} X_{j}=0
$$

Так как $X-$ комультипликационный модуль, то существует такое множество идеалов $\left\{B_{i}\right\}_{i \in I}$ кольца $A$, что

$$
X_{i}=\ell_{X}\left(B_{i}\right)
$$

для любого $i \in I$. Обозначим через $B$ идеал $\sum_{i \in I} B_{i}$ кольца $A$. Тогда

$$
\ell_{X}(B)=\ell_{X}\left(\sum_{i \in I} B_{i}\right)=\bigcap_{i \in I} \ell_{X}\left(B_{i}\right)=\bigcap_{i \in I} X_{i}=0 .
$$

Из утверждения 3 леммы 12 вытекает, что $B=A$. Тогда $1 \in \sum_{i \in I} B_{i}$. Поэтому существует конечное подмножество $J$ в $I$, удовлетворяющее условию

$$
B=\sum_{j \in J} B_{j}
$$


Тогда

$$
\bigcap_{j \in J} X_{j}=\bigcap_{j \in J} \ell_{X}\left(B_{j}\right)=\ell\left(\sum_{j \in J} B_{j}\right)=\ell_{X}(A)=0 .
$$

Пятое утверждение вытекает из четвертого.

Докажем шестое утверждение. Пусть $B$ - максимальный идеал кольца $A$, содержащий $r_{A}(X)$. Если $\ell_{X}(B)=0$, то в силу первого утверждения

$$
A=B+r_{A}(X)=B,
$$

и получаем противоречие. Поэтому $\ell_{X}(B) \neq 0$. В силу части 2 леммы $10, \ell_{X}(B)-$ простой модуль. В силу четвертого утверждения, $\ell_{X}(B)$ совпадает с одним из модулей $X_{1}, \ldots, X_{n}$. Поэтому идеал $B$ совпадает с одним из идеалов $r_{A}\left(X_{1}\right), \ldots, r_{A}\left(X_{n}\right)$, и кольцо $A / r_{A}(X)$ имеет только конечное число максимальных правых идеалов.

Предложение 1. Пусть A-регулярное кольцуо, в котором все идемпотенты центральны, и М - правый А-модуль. Тогда следующие условия равносильны:

(1) $М$ - комультипликацчионный правый А-модуль;

(2) либо $M$ прост, либо

$$
M=\bigoplus_{i \in I} M_{i}, \quad|I|>1,
$$

где все $M_{i}$ - попарно неизоморфные простые модули, $и$

$$
\bigcap_{j \neq i} r_{A}\left(M_{j}\right) \nsubseteq r_{A}\left(M_{i}\right)
$$

для каждого $i \in I$.

Доказательство. Хорошо известно, что $A-$ инвариантное кольцо, над которым все модули полупримитивны (см., например, $[15,7.2,22.3,22.1])$. Поэтому импликация (2) $\Rightarrow(1)$ следует из леммы 11 , а импликация (1) $\Rightarrow(2)$ вытекает из леммы 8.

Доказательство теоремь 1. Первое утверждение вытекает из утверждений 4, 5 и 6 леммы 12.

Докажем второе утверждение. В силу утверждения 4 леммы 12, можно считать, что каждый ненулевой конечнопорожденный подмодуль в $M$ является существенным расширением конечной прямой суммы простых модулей. В силу леммы 8, можно считать, что модуль $M$ полупрост. Теперь искомое утверждение вытекает из леммы 11.

Третье утверждение вытекает из предложения 1.

\section{Список литературы}

1. Al-Shaniafi Y., Smith P. F., Comultiplication modules over commutative rings. J. Commutative Algebra (2011) 3, 1-29. 
2. Ansari-Toroghy H., Farshadifar H., The dual notion of multiplication modules. Taiwanese J. Math. (2007) 11, 1189-1201.

3. Ansari-Toroghy H., Farshadifar H., Comultiplication modules and related results. Honam Math. J. (2008) 30, 91-99.

4. Ansari-Toroghy H., Farshadifar H., On comultiplication modules. Korean Ann. Math. (2008) 25, $57-66$.

5. Ansari-Toroghy H., Farshadifar H., On endomorphisms of multiplication and comultiplication modules. Arch. Math. (2008) 44, 9-15.

6. Ansari-Toroghy H., Farshadifar H., Strong comultiplication modules. CMU. J. Nat. Sci. (2009) 8, $105-113$.

7. Ansari-Toroghy H., Farshadifar H., On multiplication and comultiplication modules. Acta Math. Sci. (2011) B31, 694-700.

8. Ansari-Toroghy H., Farshadifar H., On the dual notion of multiplication modules. Arabian J. Sci. Eng. (2011) 36, 925-932.

9. Ansari-Toroghy H., Farshadifar H., Mast-Zohouri M., Some remarks on multiplication and comultiplication modules. Int. Math. Forum (2009) 4, 287-291.

10. Atani R. E., Atani S. E., Comultiplication modules over a pullback of Dedekind domains. Czech. Math. J. (2009) 59, 1103-1114.

11. Atani R. E., Atani S. E., Weak comultiplication modules over a pullback of commutative local Dedekind domains. Algebra Discrete. Math. (2009) 1, 1-13.

12. Atani S. E., Saraei F. E. K., Indecomposable primarily comultiplication modules over a pullback of two Dedekind domains. Colloq. Math. (2010) 120, $23-42$.

13. Kasch F., Modules and Rings. Academic Press, London, 1982.

14. Tuganbaev A. A., Semidistributive rings and modules. Kluwer, Dordrecht, 1998.

15. Tuganbaev A. A., Rings close to regular. Kluwer, Dordrecht, 2002.

Статья поступила 22.02.2012. 